\title{
The Masters of Physics: Critical Thinking Through Skateboarding
}

\author{
Dr. William H. Robertson \\ Professor, STEM Education. Department of Teacher Education \\ The College of Education. The University of Texas at El Paso
}

\begin{abstract}
This paper focuses on the use of live action and video instruction as a method of content dissemination and to motivate students in learning in science, technology, engineering and mathematics (STEM) education. Specifically, this area, dubbed "edutainment", chronicles different interactions with students, teachers and community members that utilized live action to video as methods of capturing relevant content in student-centered contexts. The fundamental idea is to infuse one's passion into modern methods of teaching, and to inspire others to use their gifts in their education, and thereby connect them to their dreams and aspirations.
\end{abstract}

Keywords: edutainment, stem, action, science, physics, mathematics, skateboarding

\section{INTRODUCTION}

How can you get young people interested in science and mathematics? What efforts are there to integrate the experiences of middle school students into the things they need to do and learn in school? How can action sports, like skateboarding and bicycle motocross (BMX), be used to teach physics, algebra, data collection, and help students to grow in their engagement and motivation in science and mathematics?

Edutainment has recently been a major growing area of education, showing great promise to motivate students with relevant activities. The authors are among innovators who have developed cutting-edge fusions of popular culture and science, mathematics, engineering and mathematics (STEM) concepts to engage and to motivate middle school students, using vehicles such as music/song and skateboarding. The importance of using relevant and practical methods of instruction and curriculum delivery that build on student interests and increase enjoyment in the learning process are critical at the middle school level, especially in the STEM fields.

The use of edutainment in this manner is meant to inspire broader interest in mathematics and science for middle school students and to develop a culture of education that makes learning more accessible to all students. This paper surveys the use of such immersive modalities (including some specific vignettes) to involve students and teachers actively with concepts and suggests further directions for the use of demonstrations and videos in educational settings.

Throughout history, there have been abundant examples of entertainment education (also called by its portmanteau, edutainment), especially for health and social issues (e.g., Singhal, Wang, \& Rogers, 2012). The purpose of this article is to focus on video edutainment in the specific context of STEM education, chronicling large-scale interactions (as opposed to, for example, digital games individuals can play) with students, teachers and community members involving relevant video content in student-centered contexts. 
The focus on edutainment through video is these examples is on examples related skateboarding, where the entertainment value is significant, but not overshadowing the educational content. This is also in agreement with Resnick (1987) to avoid implying that education is a "bitter medicine that needs the sugar-coating of entertainment to become palatable" or that education and entertainment are "services that someone else provides for you" rather than "things that you do."

From my work with students in K-12, with whom I have regular interactions due to the fieldbased nature of my work in the College of Education, I have come to see that students access information in new ways that differ from methods we usually use to deliver content to students. For example, while many learners in my generation have grown up accessing textual information, the use of Google as an Internet search engine to find articles or relevant textual content is quite normal. Yet, for those students currently in middle school, their first step is often to look for a video with content on YouTube and then once they have seen and heard something about a topic, then go and explore it with text and actual experiences. This is the type of modern learner to which I am appealing, as well as to assist the teachers with whom they are working with in schools.

The use of action science as a method for integrating transformative education is an approach that appears to be enhancing the interest and motivation of students in science. It is the purpose of Dr. Skateboard's Action Science to positively impact achievement for middle and high school students in the area of physical science knowledge and skills. By immersing students in a science learning approach that is based on action sports and focuses on the goals and objectives in physical science, the process skills and overall content knowledge of the students have the potential to greatly increase. Studies have shown that students, who are involved in active learning in meaningful contexts, acquire knowledge and become proficient in problem solving. The long-term prospects of this approach seeks to determine how the implementation of curriculum approaches built around student interests such as skateboarding and bicycle motocross (BMX) can impact student achievement in the area of science content and conceptual understandings.

\section{PARTICULAR NEED FOR MOTIVATION THROUGH EDUTAINMENT IN STEM}

The use of strategies of edutainment in science and mathematics as a mechanism for integrating transformative education is an approach that appears to be enhancing the interest and motivation of middle school students in science. It is the purpose of immersive and entertaining educational opportunities to positively impact achievement for middle school students in STEM, especially in the areas of science and mathematics knowledge and skills. By immersing students in a learning approach that is based on appealing delivery of content and focuses on the goals and objectives in middle school science and mathematics, the process skills and overall content knowledge of the students have the potential to greatly increase. Studies have shown that students, who are involved in active learning in meaningful contexts, acquire knowledge and become proficient in problem solving (Author, 2008). The long-term prospects of this research area will seek to determine how the implementation of curriculum approaches integrating strategies in edutainment and built around student interests such as skateboarding and music can impact student achievement in the area of science and mathematics content and conceptual understandings.

Each learner understands content and concepts differently based on his or her previous experiences, and the materials help to provide a context for understanding both science concepts and real world connections. So much fascinating content is at the fingertips of 
learners everywhere, and with computer access and technology becoming more affordable, more information is accessible. The main emphasis is to engage students in the exploration of STEM topics in a real-world context and to link education to delivery methods that integrate entertainment value and presentation. The students need opportunities to address misconceptions and to develop concepts in real world situations. "Students come to school with their own ideas, some correct and some not, about almost every topic they are likely to encounter" (Rutherford \& Algren, 1990, p. 198). Learning is the responsibility of the learner, but the teacher guides the student into developing meaning from content material and classroom experience.

Learning happens when you go to areas of high risk and high ambiguity. Yet it's not just enough to learn -- the goal should really center on mastery. To master something takes a long time, which skateboarders at a local park know fundamentally. My years in the field of education continually reinforce my belief that it's less about what you know and more about what you can master. When you master something, you know what it takes to be successful, and then you can apply that ability to other aspects of your life. Whether a student is mastering skateboarding, painting, the guitar, a new language, science, or mathematics, developing one kind of mastery can help him or her master something else. For teachers, we need to inspire others to use their gifts in their education, and thereby connect them to their dreams and aspirations.

In the classroom, constructivist curriculum must be designed so that it reflects real life situations (Bentley, 1995), and the use of relevant contexts helps to contextualize the concepts, as well as help provide connections across subject areas (Hofstein \& Yager, 1982). Research scientists and mathematicians cross over the barriers between academic disciplines all the time, and seldom operate solely on isolated areas of content, but integrate the use of language, knowledge and process application. STEM programs that emphasize investigation give students the ability to retain facts through critical thinking by working through problems logically and making connections to the real world.

It is important to engage learners in learning situations that effectively integrate their own experiences and familiar materials that students can use to better understand specific concepts, especially in the STEM fields (Eisenkraft, 2003). For example, students who enjoy skateboarding can be given opportunities to explore the concepts of velocity, acceleration, center of gravity, and moment of inertia. They may also use the skateboard and a local skatepark to investigate topics such as inclined planes, levers, fulcrums and screws. The purpose of this approach is to allow the students to explore meaningful science topics set in the context of something they enjoy doing.

\section{AN EXAMPLE: SCIENTIFIC SKATEBOARDING}

Dr. Skateboard's Action Science is a curriculum supplement for middle school (6-8) students that is designed to address content and process objectives in physical science for the Texas Essential Knowledge and Skills (TEKS). The video instruction and twenty classroom activities provide the teacher with a series of instructional tools and content information that can be used to explore and explain the concepts found in the areas of forces, motion, Newton's Laws of Motion, and simple machines. It is the purpose of this research project to determine what impact Dr. Skateboard's Action Science has on a sample of Middle School students in the area of physical science knowledge and skills.

Dr. Skateboard's Action Science maps to the physical science TEKS in which all middle school students need to be engaged. Dr. Skateboard's Action Science explores scientific concepts in a 
curriculum that is designed to address both physical science content and process skills. The video instruction focuses on the physical science concepts found in the areas of motion, forces, Newton's Laws of Motion, and simple machines. The main purpose is to provide an interesting method of engaging students in the exploration of science in a real-world context. The overarching theme for Dr. Skateboard's Action Science is the appeal of action sports as teaching and learning vehicles for students, teachers, and the community.

Dr. Skateboard's Action Science is an example of transformative education, a student-centered curriculum supplement built around interesting content linked to specific physic knowledge and skills in science. The videos and classroom materials provide the classroom teacher with an instructional series rich in science and including topics such as centrifugal and centripetal forces, inertia, center of gravity, and momentum. The purpose is to contextualize the classroom process of acquiring critical knowledge, developing proficiency in problem solving, engaging in self-directed learning, and participating in collaborative teams.

The activities and materials are designed for students to interact in small teams, and this sharing within cooperative groups is a fundamental constructivist strategy that allows the teacher to facilitate the learning process. As a student-centered approach, it also helps to develop a common base of experiences on which to help students make connections to content. In the classroom, problem-solving strategies depend on the development of conceptual understandings, and hands-on explorations of simple topics combined with collaborative interactions among learners help to build an understanding of processes and concepts (Apple, 1993). It is important for educators to not merely regard the learner's point of view alone as fully complete and significant (Dewey, 1970), but to guide the students in the analysis and synthesis of content information. The learner is always defining meaning within the context of action and reflection (Brooks \& Brooks, 1993), and the social situations, including discussion, explanations and hands-on experiences, provide the context for knowledge construction.

The video segments themselves do provide action, but also relevant content for the classroom, and complement the activities that teachers can implement in the classroom, and in tandem, can help reinforce the conceptual emphasis in a lesson. For example, the teacher can utilize the portion of the "Newton's Laws" video that covers the concepts of force, mass and acceleration, which is designed as an effective introduction to the activity "Force Makes a Mass Move". This brief video segment serves as a hook in order to introduce the activity and additionally as a review for the content covered in class. In that sense, the materials serve both pre-activity and post-activity purposes, and allow the teacher the flexibility to have students explain fundamental physics as well as pursue inquiry extensions. Each activity contains both a teacher section and a student section. The teacher section provides standards alignment information, background knowledge, guiding questions with answers and extensions for student enrichment. The student section contains the classroom science activity, connections to real world examples, explanations of concepts and actual photographs of BMX riders and skateboarders in action.

Action Science is designed to teach fundamental science concepts in physics in an approach that utilizes transformative educational strategies, which help students move from memorizing facts and content to constructing knowledge in meaningful and useful manners. The activities associated with Action Science address both the objectives and enduring knowledge of physical science in content and process skills for both the United States of America (USA) National Science Standards and the Texas Essential Knowledge and Skills (TEKS) state standards. 
The materials in Dr. Skateboard's Action Science were also designed to emphasize inquiry in classroom explorations. As a foundation for discovery, the teacher can use the video segment in the "Simple Machines" episode that relates to fulcrums and levers, and then have the students perform the classroom activity "Skateboards Have Levers and Fulcrums." After the activity, the teacher may revisit these ideas and then create an extension inquiry exercise for the students to do in teams. The teacher can provide the students with the same materials used in the activity such as rulers, tape, plastic spoons, rubber bands, and modelling clay and challenge the students to design a simple machine made of at least three of the provided that uses a lever and a fulcrum and can propel a small marshmallow the farthest distance.

In making this transition in class, the teacher guides the students towards developing their own ideas and within a given time period, has the students create and test their unique designs. By engaging students in a design competition, there is a spirit of enthusiasm and excitement among the groups. There are also excellent opportunities to develop cooperative group skills and to have students use critical thinking to solve the problem presented. "Students should know what it feels like to be completely absorbed in a problem. They seldom experience this feeling in school" (Bruner, 1962, p. 50). Finally, the teams of students have to not only launch the marshmallow, but they also have to record the distances, calculate the average distances travelled and identify the lever and fulcrum within their machine. In this manner, the students have to present their ideas, justify their understandings and support their findings with experimental data.

Another classroom example in the design of the series is the use of the video segment in the "Forces" video that focuses on the concept of center of gravity, which additionally bridges the concepts of gravity and lift. Prior to showing the video segment, the teacher can use openended questions with students in order to activate their previous knowledge concerning this content. Sample questions could include, "What do you do when you ride a skateboard or a bicycle?", "How do you balance on a skateboard or bike?" and "What forces are acting on you as you are trying to ride a bike or skateboard?" Additionally, previously marginalized students who have experience in these activities, but may struggle in science, can become experts in this discussion and contribute greatly to the classroom investigations.

Finally, the teacher should conclude the series of questions by asking, "What is the center of gravity and why is it important?", and then facilitate the conversation in order to introduce the segment in the "Forces" video that covers gravity, lift and the center of gravity. This approximately 4-minute segment of the video then serves as the engagement to the activity "Flatland BMX and the Center of gravity" in which students create irregular cardboard shapes and determine the object's center of gravity through a series of step-by-step procedures. Students exploring a concept should be given opportunities to work with hands-on materials so that they can have experiences that are real and fundamental. Hands-on learning plays a valuable role in the constructivist paradigm, as it is the process of learning by doing (Dewey, 1970) that is utilized in explorations and experiments.

Next, students modify their shapes either by adding paper clips (which increases the mass) or by cutting off part of some of cardboard (which decreases the mass). In turn, they come to see that there is a fundamental relationship between the center of gravity and the mass of an object, and that the center of gravity will move in relation to an increase or a decrease in mass. After the classroom lesson, the teacher can revisit the activity by asking the students to explain their findings and the relationships they discovered. As students explore concepts, they develop a broader understanding of those concepts. When they relate what they are learning, seeing or doing to others, they can begin to see similarities in their understandings, as well as 
self identify misconceptions they may have about content material (Bybee, 2006). Finally, there is a list of open-ended questions for students to answer, as well as extensions that they can engage in if there is additional time and motivation to explore these concepts. This entire activity can be done in the timeframe of a normal class period with minimal setup and cleanup, and can provide both teachers and students an interesting alternative to exploring these fundamental physics ideas.

\section{CONCLUSIONS}

The use of educational materials contextualized in the form of entertainment has a long history, and has certainly been gaining momentum as delivery methods of content have grown to utilize multimedia and video. The connection of STEM education to real world topics is vital in order to engage students effectively and to provide them with a reason to delve into deeper conceptual understandings. Edutainment, and its synthesis of elements of education and entertainment, has great potential at the middle school level to serve as a primary motivational and engagement strategy for STEM efforts, especially in the STEM areas. Additionally, the potential to reach wider audiences in STEM utilizing edutainment strategies can help to transform STEM education by integrating both informal and formal learning in ways that increase student interest and inspire learners to pursue STEM in school and, ultimately, as professions.

\section{References}

Apple, M. W. (1993). Official knowledge. New York: Routledge.

Bentley, M. L. (1995). Carpe diem. Science activities, 32(3), 23-30.

Brooks, J. G. and Brooks, M.G. (1993). The case for the constructivist classroom. Alexandria, VA: ASCD Press. Bruner, J. (1962). The process of education. Cambridge: Harvard University Press.

Bybee, R. W. et al (2006). The BSCS 5e instructional model: Origins, effectiveness, and applications (Executive Summary). [On-Line] Accessed September 9, 2007 at http://www.bscs.org/pdf/bscs5eexecsummary.pdf

Dewey, J. (1970). The child and the curriculum. Chicago: Chicago University Press

Eisenkraft, A. (2003). Expanding the 5E Model. The Science Teacher. 70(6), 57-59.

Hofstein, A. and Yager, R. (1982). Societal issues as organizers for science education in the 80s. School Science and Mathematics, 82(15), 539-547.

Resnick, M. (1987). Edutainment? No thanks. I prefer playful learning. Associazione Civita Report on Edutainment, pp. 1-4.

Author (2008). Developing problem-based curriculum: Unlocking student success utilizing critical thinking and inquiry, Des Moines, IA: Kendall Hunt Publishing.

Author (2008). Dr. Skateboard's Action Science [Disc 2]. Tempe, AZ: Day Publishers.

Rutherford, F. J. and Algrehn, A. (1990). Science for all Americans. New York: Oxford University Press.

Singhal, A., Wang, H., and Rogers, E. M. (2012). The rising tide of entertainment-education in communication campaigns. In R. E. Rice and C. K. Atkin (Eds.), Public communication campaigns (4th ed.) (pp. 323-335). Beverly Hills, CA: Sage. 